\title{
Transgenic expression of delta- 6 and delta-15 fatty acid desaturases enhances omega-3 polyunsaturated fatty acid accumulation in Synechocystis sp. PCC6803
}

Gao Chen ${ }^{1,2}$, Shujie Qu ${ }^{5}$, Qiang Wang ${ }^{4}$, Fei Bian ${ }^{1,2}$, Zhenying Peng ${ }^{1,2}$, Yan Zhang ${ }^{1,2}$, Haitao Ge ${ }^{6}$, Jinhui Yu ${ }^{1,2}$, Ning Xuan ${ }^{1,2}$, Yuping $\mathrm{Bi}^{1,2^{*}}$ and Qingfang $\mathrm{He}^{1,2,3^{*}}$

\begin{abstract}
Background: Polyunsaturated fatty acids (PUFAs), which contain two or more double bonds in their backbone, are the focus of intensive global research, because of their nutritional value, medicinal applications, and potential use as biofuel. However, the ability to produce these economically important compounds is limited, because it is both expensive and technically challenging to separate omega-3 polyunsaturated fatty acids ( $\omega-3$ PUFAs) from natural oils. Although the biosynthetic pathways of some plant and microalgal $\omega-3$ PUFAs have been deciphered, current understanding of the correlation between fatty acid desaturase content and fatty acid synthesis in Synechocystis sp. PCC6803 is incomplete.

Results: We constructed a series of homologous vectors for the endogenous and exogenous expression of $\Delta 6$ and $\Delta 15$ fatty acid desaturases under the control of the photosynthesis psbA2 promoter in transgenic Synechocystis sp. PCC6803. We generated six homologous recombinants, harboring various fatty acid desaturase genes from Synechocystis sp. PCC6803, Gibberella fujikuroi and Mortierella alpina. These lines produced up to $8.9 \mathrm{mg} / \mathrm{l}$ of a-linolenic acid (ALA) and $4.1 \mathrm{mg} / \mathrm{l}$ of stearidonic acid (SDA), which are more than six times the corresponding wild-type levels, at $20^{\circ} \mathrm{C}$ and $30^{\circ} \mathrm{C}$. Thus, transgenic expression of $\Delta 6$ and $\Delta 15$ fatty acid desaturases enhances the accumulation of specific $\omega-3$ PUFAs in Synechocystis sp. PCC6803.

Conclusions: In the blue-green alga Synechocystis sp. PCC6803, overexpression of endogenous and exogenous genes encoding PUFA desaturases markedly increased accumulation of ALA and SDA and decreased accumulation of linoleic acid and $y$-linolenic acid. This study lays the foundation for increasing the fatty acid content of cyanobacteria and, ultimately, for producing nutritional and medicinal products with high levels of essential $\omega-3$ PUFAs.
\end{abstract}

Keywords: Omega-3 polyunsaturated fatty acids, Gene expression, Fatty acid desaturase, Synechocystis sp. PCC6803

\section{Background}

Polyunsaturated fatty acids (PUFAs) are hydrocarbon chains that are 18 to 22 carbons in length, and have two or more double bonds in their backbone structure. PUFAs are classified as omega-3 ( $\omega-3)$ and omega-6 ( $\omega-6)$ fatty acids (FAs), based on the position of the first double bond from the methyl end. PUFAs, especially $\omega-3$ PUFAs,

\footnotetext{
*Correspondence: yuping_bi@hotmail.com; qfhe@ualr.edu

'Biotechnology Research Center, Shandong Academy of Agricultural

Sciences, Jinan 250100, P. R. China

${ }^{2}$ Shandong Provincial Key Laboratory of Genetic Improvement, Ecology and Physiology of Crops, Jinan 250100, P. R. China

Full list of author information is available at the end of the article
}

are essential dietary molecules with potential medicinal applications, and have recently become the focus of intensive research. The importance of $\omega-3$ PUFAs in disease prevention and human nutrition has been scientifically recognized for a number of years. Intake of $\omega-3$ PUFAs is reported to reduce the risk of cardiovascular disease [1-4], neurological disorder [5], inflammation [6-8], and cancer [9]. Stearidonic acid (SDA, 18:4n-3) is a highly unsaturated plant-based $\omega-3$ PUFAs with potential health benefits. SDA is a metabolic intermediate in the conversion of $\alpha$-linolenic acid (ALA, 18:3n-3) to eicosapentaenoic acid (EPA, 20:5n-3) and docosahexaenioc acid (DHA, 22:6n-3),

\section{Biomed Central}


and is more stable than either EPA or DHA because of its lower unsaturation index. Because SDA is readily converted into EPA and DHA upon consumption, it may potentially be used to increase blood levels of $\omega-3$ PUFAs $[10,11]$. Indeed, ingestion of vegetable oils enriched in SDA has been shown to increase EPA concentrations in tissue [12], and Kawabata et al. [13] demonstrated that increasing the consumption of SDA-containing soybean oil modifies the lipid and FA profiles in body fats. Becasue $\omega-6$ PUFAs are not readily converted into $\omega-3$ PUFAs, the ratio of $\omega-6$ to $\omega-3$ PUFAs is largely determined by dietary intake of FAs [14]. Modern diets tend to contain excessive levels of $\omega-6$ FAs, such as linoleic acid (LA) and $\gamma$ linolenic acid (GLA), and low levels of $\omega-3$ FAs, such as ALA and SDA [15]. Ancestral dietary fat compositions exhibited a $\omega-6$ to $\omega-3$ ratio of $2: 1$ to $4: 1$, but this ratio can be as high as 10:1 in modern diets [16]. These imbalances can increase the risk of hypertension [17], cardiovascular disease [18], rheumatoid arthritis [19-21], and inflammatory and autoimmune disease [22,23].

PUFAs also have potential applications in biofuel production. Genetic and metabolic engineering techniques can be used to increase targeted lipid content (specific or total lipid), and reduce the cost of microalgal diesel production [24-26]. The FA composition of the oil determines the storage stability of biodiesel [27]. Although PUFAs are more susceptible to oxidation, a higher percentage of unsaturated FAs in the feedstock oil results in biodiesel with improved cold-flow properties [28]. Therefore, research that aims to improve production of PUFAs will have a positive impact on food security, human nutrition, and biodiesel production.

Traditionally, $\omega-3$ PUFA products have been derived mainly from fish oil and shellfish [29,30]. However, overfishing has seriously depleted resources [31], and it is expensive and technically challenging to extract $\omega-3$ PUFAs from their natural sources. There is thus an urgent need to identify alternative and sustainable sources of $\omega$-3 PUFAs [32-34]. Oils are mainly composed of palmitic acid (PA, C16:0), oleic acid (OA, C18:1n-9), ALA, and SDA $[35,36]$, and enhancing the production of these individual FAs would improve oil production.

Metabolic engineering provides a powerful and effective approach for enhancing production of PUFAs. The FA biosynthetic pathways in higher plants and microalgae have been well studied, and much research has recently focused on developing metabolic engineering methods to increase the FA content of microalgae [37-41] and oilseed crops [42,43]. Several groups have generated transgenic plants that synthesize and accumulate PUFAs in storage seed oils [44-48]. Reddy and Thomas used the constitutive Cauliflower Mosaic Virus (CaMV) 35S promoter to control the expression of a $\Delta 6$ desaturase (isolated from the cyanobacterium Synechocystis) in Nicotiana tabacum (tobacco plant), which resulted in accumulation of low levels of GLA and SDA in the transgenic leaves [49]. Much higher levels of GLA and SDA accumulation (accounting for $20 \%$ of total FAs) were obtained by expressing the $\Delta 6$ desaturase from Borago officinalis in tobacco [50] and oilseed crops [51]. Meanwhile, the biotechnology companies Monsanto and Solae LLC successfully bred transgenic soybean plants producing 5 to $8 \%$ GLA and 15 to $30 \%$ SDA. These transgenic soybean plants have now been commercialized after passing a safety assessment [52]. Ruiz-López et al. [53] expressed a $\Delta 6$ desaturase from Primula vialii in linseed, and the transgenic plants accumulated $13.4 \%$ SDA without any GLA in their lipids. More recently, transgenic plants producing EPA and DHA, generated by various approaches, have been reported by several groups; however, the levels of PUFAs achieved are not nearly as high as those in fish oil. Other attempts to increase PUFA content in transgenic plants include optimization of various aspects, such as identification of improved desaturases or of the specific acyl-exchange mechanisms of PUFAs, maintenance of a continuous flux of substrates through the PUFA biosynthesis pathways without significant negative influence on triacylglycerol (TAG), and co-expression of transgenes $[14,54]$.

Cyanobacteria are prokaryotes that are able to produce valuable metabolites using energy from sunlight. The blue-green alga Synechocystis sp. PCC6803, a facultative phototroph, is a unicellular cyanobacterium that is ideal for studying FA accumulation, and it has been explored as a vector for commercial manufacture of biofuels and oils [55]. In this study, we cloned the $\Delta 6$ and $\Delta 15 \mathrm{FA}$ desaturases of Synechocystis sp. PCC6803, optimized the codon usage of the $M$. alpina $\triangle 6$ FA desaturase and the $G$. fujikuroi bifunctional $\Delta 12 / \Delta 15$ FA desaturase for optimal translation in Synechocystis sp. PCC6803, and expressed these genes in Synechocystis sp. PCC6803. We then analyzed the FA content and composition by gas chromatography (GC), and determined the correlations between the variety of FA desaturase and FA synthesis. We also investigated the expression patterns of endogenous and exogenous $\Delta 6$ and $\Delta 15 \mathrm{FA}$ desaturases at $20^{\circ} \mathrm{C}$ and $30^{\circ} \mathrm{C}$, respectively, using quantitative real-time PCR and immunoblot analysis. By overexpression of endogenous and exogenous genes encoding PUFA desaturases, we markedly increased the accumulation of ALA and SDA and decreased the accumulation of LA and GLA in this cyanobacterium.

\section{Results and discussion}

Transgenic expression of $\Delta 6$ and $\Delta 15$ genes in Synechocystis

Transformants were selected by subculture on BG-11 solid medium containing kanamycin $50 \mu \mathrm{g} / \mathrm{ml}$. The complete 
segregation of the transformants was confirmed by PCR (Figure 1A). Amplification of the $\Delta 15$ or $\Delta 6$ gene fragment with the $p s b A 2$ promoter, T1T2 terminator, and kanamycin cassette, coupled with the lack of amplification of the DNA fragment $(1.5 \mathrm{~kb})$ of the wild-type (WT) psbA2 gene and promoter, indicated that the Synechocystis lines were indeed the expected transformants.

To detect the expression of $\Delta 15$ or $\Delta 6$ in transformants, we exposed the WT and $\Delta 6 / \Delta 15$ transformant cells to temperatures of $30^{\circ} \mathrm{C}$. Immunoblot analysis confirmed the expression of the $\Delta 6$ and $\Delta 15$ desaturases, cloned in-frame with the psbA2 promoter and T1T2 terminator, in the Synechocystis transformants. Immunoblot analysis of the $\Delta 6$ and $\Delta 15$ desaturases using His tag and Flag tag antibodies, respectively, confirmed the presence of the $\Delta 6$ and $\Delta 15$ proteins in Synechocysits transformants (Figure $1 \mathrm{~B}$ ). The $\Delta 6$ and $\Delta 15$ proteins localized to the soluble fraction of Synechocystis cell extracts.

\section{ALA and SDA production by transgenic cyanobacterial cells}

The physicochemical properties of the cell membrane are reported to change rapidly with changes in temperature [44-47]. To study the effect of temperature on FA content, WT and mutant Synechocystis sp. PCC6803 cells were separately grown under mixotrophic conditions at 20 and $30^{\circ} \mathrm{C}$ and then the FA content in membranes was analyzed by GC (Table 1 ).

In WT cells grown under mixotrophic conditions at $30^{\circ} \mathrm{C}$, the total FA content was about $75.20 \mathrm{mg} / \mathrm{g}$ (dry weight). Compared with the WT, the FA content of transgenic Synechocystis sp. PCC6803 overexpressing Synechocystis $\Delta 15$ desaturase increased by $32.5 \%$. In WT cells grown under mixotrophic conditions at $20^{\circ} \mathrm{C}$, the total FA content was about $60.64 \mathrm{mg} / \mathrm{g}$, but overexpression of Synechocystis $\Delta 15$ desaturase (SDSy15 vector) resulted in a slight increase in total FA content (0.2\%). Compared with WT (see Additional file 1: Figure S2A), the C18:1, C18:2, and C18:3n- 6 content of the transgenic cells was markedly lower, and the C18:3n-3 and C18:4 content markedly higher. For example, under mixotrophic cultivation at $30^{\circ} \mathrm{C}, \mathrm{C} 18: 3 \mathrm{n}-3$ increased from $1.45 \%$ to $17.52 \%$ and $\mathrm{C} 18: 4$ increased from $1.20 \%$ to $9.11 \%$ (see Additional file 1: Figure $\mathrm{S} 2 \mathrm{~B}$ ), whereas at $20^{\circ} \mathrm{C} \mathrm{C} 18: 3 \mathrm{n} 3$ increased from $2.23 \%$ to $23.05 \%$ and $\mathrm{C} 18: 4$ increased from $1.54 \%$ to $10.77 \%$ (see Additional file 1: Figure S2A', Figure S2B'). In our experiments, from $1000 \mathrm{ml}$ of BG-11 medium under mixotrophic cultivation at $20^{\circ} \mathrm{C}$, we obtained $0.63 \mathrm{~g}$ (dry weight) of Synechocystis cells overexpressing $\Delta 15$ desaturase. GC analysis showed that this line produced up to $8.9 \mathrm{mg} / \mathrm{l}$ and $4.1 \mathrm{mg} / \mathrm{l}$ of ALA and SDA, respectively. These results suggest that overexpression of Synechocystis $\Delta 15$ desaturase in Synechocystis sp. PCC6803 improves

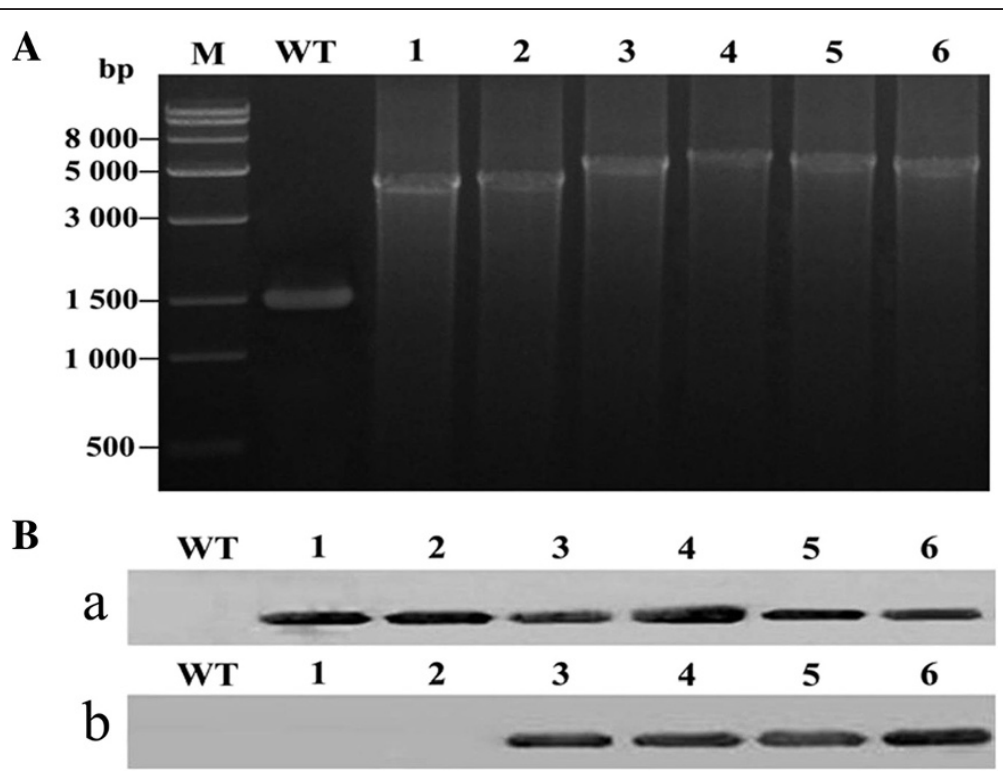

Figure 1 PCR and immunoblot analysis of wild-type (WT) Synechocystis and desaturase transformants. (A) PCR analysis of transgenic Synechocystis, in which a fragment of psbA2 was deleted and replaced with various exogenous genes. M, Trans15k DNA marker; WT, wild-type Synechocystis sp. PCC6803; lane 1, pSDSy15; lane 2, pSDGf1215; lane 3, pSDSy15Sy6; lane 4, pSDGf1215Ma6; lane 5, pSDSy15Ma6; lane 6, pSDGf1215Sy6. The primers used in the PCR analysis (psbA2 promoter- $F$ and psbA2-R) are described in the Methods section, and were combined to amplify the psbA2 fragments (1.5 kb). (B) Immunoblot analysis of WT Synechocystis and desaturase transformants using (a) Flag tag and (b) His tag antibodies. Lane 1, WT Synechocystis sp. PCC6803; lanes 2-7, pSDSy15, pSDGf1215, pSDSy15Sy6, pSDGf1215Ma6, pSDSy15Ma6, and pSDGf1215Sy6 Synechocystis transformants, respectively. 
Table 1 Fatty acid content of wild-type and transgenic Synechocystis sp. PCC6803 ${ }^{\mathrm{a}-\mathrm{d}}$

\begin{tabular}{|c|c|c|c|c|c|c|c|c|}
\hline \multirow[t]{2}{*}{ Strain } & \multirow[t]{2}{*}{$\mathrm{T},{ }^{\circ} \mathrm{C}$} & \multirow[t]{2}{*}{$\mathrm{TFA}, \mathrm{mg} / \mathrm{g}$} & \multicolumn{6}{|c|}{ FA content, $\%$} \\
\hline & & & C18:1 & C18:2 & C18:3n-6 & C18:3n-3 & C18:4 & Others \\
\hline \multirow[t]{2}{*}{ Wild type } & 30 & 75.201 & $7.07 \pm 0.9$ & $13.59 \pm 1.0$ & $16.26 \pm 1.2$ & $1.45 \pm 0.2$ & $1.20 \pm 0.3$ & $60.44 \pm 4.3$ \\
\hline & 20 & 60.640 & $3.94 \pm 0.2$ & $16.75 \pm 0.6$ & $14.72 \pm 1.3$ & $2.53 \pm 0.3$ & $1.54 \pm 0.2$ & $60.53 \pm 3.6$ \\
\hline \multirow[t]{2}{*}{ pSDSy 15} & 30 & 50.755 & $2.73 \pm 0.3$ & $2.80 \pm 0.5$ & $0.30 \pm 0.1$ & $17.52 \pm 2.3$ & $9.11 \pm 1.3$ & $67.54 \pm 7.3$ \\
\hline & 20 & 60.760 & $3.27 \pm 0.7$ & $1.94 \pm 0.3$ & $0.23 \pm 0.1$ & $23.05 \pm 2.3$ & $10.77 \pm 1.6$ & $60.73 \pm 5.7$ \\
\hline \multirow[t]{2}{*}{ pSDGf1215 } & 30 & 67.085 & $3.17 \pm 0.3$ & $15.56 \pm 1.3$ & $13.79 \pm 2.0$ & $1.78 \pm 0.3$ & $1.20 \pm 0.2$ & $64.50 \pm 5.5$ \\
\hline & 20 & 66.990 & $5.73 \pm 1.3$ & $17.67 \pm 1.0$ & $12.71 \pm 0.8$ & $1.63 \pm 0.1$ & $0.96 \pm 0.2$ & $61.29 \pm 8.3$ \\
\hline \multirow[t]{2}{*}{ pSDSy15Sy6 } & 30 & 63.071 & $4.10 \pm 0.8$ & $2.57 \pm 0.6$ & $0.21 \pm 0.1$ & $23.64 \pm 3.4$ & $7.76 \pm 0.7$ & $61.72 \pm 8.1$ \\
\hline & 20 & 57.130 & $2.28 \pm 0.2$ & $1.30 \pm 0.3$ & $0.19 \pm 0.1$ & $16.35 \pm 1.9$ & $13.12 \pm 1.3$ & $66.77 \pm 6.5$ \\
\hline \multirow[t]{2}{*}{ pSDGf1215M6 } & 30 & 68.803 & $3.11 \pm 0.4$ & $17.21 \pm 2.3$ & $14.45 \pm 1.6$ & $2.05 \pm 0.2$ & $1.18 \pm 0.1$ & $61.98 \pm 5.3$ \\
\hline & 20 & 75.657 & $1.20 \pm 0.1$ & $10.65 \pm 2.3$ & $17.16 \pm 1.6$ & $3.02 \pm 0.5$ & $1.70 \pm 0.1$ & $66.27 \pm 5.9$ \\
\hline \multirow[t]{2}{*}{ pSDSy15Ma6 } & 30 & 61.480 & $3.45 \pm 0.2$ & $0.70 \pm 0.1$ & $0.21 \pm 0.1$ & $17.79 \pm 2.3$ & $11.10 \pm 1.5$ & $66.76 \pm 3.9$ \\
\hline & 20 & 35.190 & $2.03 \pm 0.2$ & $1.22 \pm 0.3$ & $0.14 \pm 0.1$ & $14.83 \pm 2.9$ & $12.36 \pm 1.5$ & $69.42 \pm 5.3$ \\
\hline \multirow[t]{2}{*}{ pSDGf1215Sy6 } & 30 & 59.192 & $2.80 \pm 0.2$ & $15.98 \pm 0.6$ & $13.95 \pm 0.7$ & $2.40 \pm 0.4$ & $1.57 \pm 0.1$ & $63.32 \pm 6.0$ \\
\hline & 20 & 53.651 & $6.75 \pm 1.2$ & $17.17 \pm 2.0$ & $10.81 \pm 0.9$ & $2.11 \pm 0.6$ & $0.89 \pm 0.1$ & $62.27 \pm 8.7$ \\
\hline
\end{tabular}

FA, fatty acid; T, Temperature; TFA, Total fatty acid.

${ }^{a}$ Values are means of triplicate experiments.

${ }^{b}$ Cells were grown under a light intensity of $40 \mu \mathrm{mol}$ photon $/ \mathrm{m}^{2} / \mathrm{s}$ for $10 \mathrm{~d}$ in BG-11 medium.

'The membrane lipids were extracted from wild-type and genetically engineered Synechocystis sp. PCC6803.

${ }^{\mathrm{d}}$ The enzymes overexpressed are indicated in parentheses (Sy15: $\Delta 15$ FA desaturase from Synechocystis sp. PCC6803; Sy6: $\Delta 6$ FA desaturase from Synechocystis sp. PCC6803; Gf1215: bifunctional $\Delta 12 / \Delta 15$ FA desaturase from Gibberella fujikuroi; Ma6: $\triangle 6$ FA desaturase from Mortierella alpina).

the yield of C18:3n-3 and C18:4, and decreases the yield of $\mathrm{C} 18: 2$ and C18:3n-6. Furthermore, the total FA content of this transformant increased when the temperature was decreased from $30^{\circ} \mathrm{C}$ to $20^{\circ} \mathrm{C}$.

Compared with the WT, the FA content also decreased in Synechocystis sp. PCC6803 lines in which Synechocystis $\Delta 6$ and $\Delta 15$ desaturases and (SDSy15Sy6 vector) or M. alpina $\Delta 6$ and Synechocystis $\Delta 15$ FA desaturase (SDSy15Ma6 vector) were tandemly expressed. The total FA content of lines transformed with SDSy15Sy6 and SDSy15Ma6 was about $63.07 \mathrm{mg} / \mathrm{g}$ and $61.48 \mathrm{mg} / \mathrm{g}$, respectively, at $30^{\circ} \mathrm{C}$, which decreased by $16.1 \%$ and $18.3 \%$ under mixotrophic cultivation, and $57.13 \mathrm{mg} / \mathrm{g}$ and $35.19 \mathrm{mg} / \mathrm{g}$ at $20^{\circ} \mathrm{C}$, which decreased by $41.9 \%$ under mixotrophic cultivation. Compared with the WT, the C18:1, C18:2, and C18:3n- 6 content decreased and the C18:3n-3 and C18:4 content markedly increased in pSDSy15Sy6 and pSDSy15Ma6. In lines transformed with SDSy15Sy6, C18:3n-3 increased from $1.45 \%$ to $23.64 \%$ and C18:4 increased from $1.20 \%$ to $7.76 \%$ (see Additional file 1: Figure S2C), while in lines transformed with SDSy15Ma6, C18:3n-3 increased from $1.45 \%$ to $17.79 \%$ and C18:4 increased from $1.20 \%$ to $11.10 \%$ (see Additional file 1: Figure S2D) under mixotrophic cultivation at $30^{\circ} \mathrm{C}$ relative to the WT control. By contrast, at $20^{\circ} \mathrm{C}$ in lines transformed with SDSy15Sy6, C18:3n-3 content increased from $2.23 \%$ to $16.35 \%$ and C18:4 increased from $1.54 \%$ to $13.12 \%$ (see Additional file 1: Figure $S 2 C^{\prime}$ ), while in lines transformed with SDSy15Ma6 under mixotrophic cultivation at $20^{\circ} \mathrm{C}$,
C18:3n-3 increased from $2.23 \%$ to $12.36 \%$ and $\mathrm{C} 18: 4$ increased from $1.54 \%$ to $14.83 \%$ (see Additional file 1 : Figure S2D').

These results show that tandem expression of $\Delta 6$ and $\Delta 15$ desaturases in Synechocystis sp. PCC6803 markedly increases the yield of C18:3n-3 and C18:4. Furthermore, C18:4 content increased as the temperature decreased, indicating that a reduction in temperature promotes the expression of $\Delta 6$ desaturase, which converts ALA to SDA. These results also suggest that production of PUFAs is greater in transgenic Synechocystis organisms cultivated at $20^{\circ} \mathrm{C}$ than in those cultivated at higher temperatures.

In addition, we generated three more types of transgenic Synechocystis sp. PCC6803, namely pSDGf1215, which overexpressed G. fujikuroi bifunctional $\Delta 12 / \Delta 15$ FA desaturase; pSDGf1215Ma6, which tandemly expressed $M$. alpina $\triangle 6$ FA desaturase and G. fujikuroi bifunctional $\Delta 12 / \Delta 15$ FA desaturase; and pSDGf1215Sy6, which tandemly expressed Synechocystis $\Delta 6$ FA desaturase and G. fujikuroi bifunctional $\Delta 12 / \Delta 15$ FA desaturase. Under mixotrophic cultivation at $30^{\circ} \mathrm{C}$, the total FA content of the lines transformed with SDGf1215, SDGf1215Ma6, and SDGf1215Sy6 was $67.09,68.80$, and $59.19 \mathrm{mg} / \mathrm{g}$, respectively, which was $10.8 \%, 8.5 \%$, and $21.3 \%$, respectively, less than that of the WT. Under mixotrophic cultivation at $20^{\circ} \mathrm{C}$, the total FA content of pSDGf 1215 and pSDGf1215Ma6 was 66.99 and $75.66 \mathrm{mg} / \mathrm{g}$, respectively, which was $10.5 \%$ and $24.8 \%$ greater than that of the WT. The total FA content of pSDGf1215Sy6 was 
$53.65 \mathrm{mg} / \mathrm{g}$, which was $11.0 \%$ less than that of the WT. Whereas the $\mathrm{C} 18: 1$ and $\mathrm{C} 18: 2$ content was much higher in the WT than in the transgenic lines, the C18:3n-6, C18:3n-3, and C18:4 content was markedly lower in the WT than in the transgenic lines.

Overexpression of endogenous $\Delta 6$ and $\Delta 15$ desaturases in Synechocystis sp. PCC6803 greatly enhanced PUFA accumulation. By contrast, heterologous expression of $M$. alpina $\triangle 6$ FA desaturase and G. fujikuroi bifunctional $\Delta 12 / \Delta 15$ FA desaturase in Synechocystis sp. PCC6803 had no obvious effect. Although both of these desaturases were previously shown to have obvious effects on FA accumulation in non-photosynthetic microbes and plants $[48,56]$, we found that overexpression of these two exogenous enzymes had no significant effect on PUFA accumulation in Synechocystis sp. PCC6803.

\section{Effects of temperature on cell growth and FA composition of transgenic Synechocystis}

Temperature has a large effect on the types of FAs produced by microalgae, and the composition of FAs, in turn, affects the physiology of the organism by changing the rate of chemical reactions and the stability of cellular components $[57,58]$. Several studies suggest that the exponential growth rates of many microalgal species increase in response to elevated growth temperatures, up to an optimal temperature, and then decline once structural integrity has been lost $[58,59]$. To preserve the structural integrity of the cell, organisms regulate lipid composition to maintain proper membrane fluidity at different temperatures [58]. In response to cold stress, several microalgal species enhance the biosynthesis and accumulation of total FAs, which also results an increase in the content of PUFAs at low temperatures [47]. To test whether overexpression of the exogenous and endogenous desaturases affects the growth and FA content of transgenic Synechocystis, we examined photoautotrophic growth and FA variation of WT and transgenic Synechocystis cells at $30^{\circ} \mathrm{C}$ and $20^{\circ} \mathrm{C}$ in liquid medium (Figure 2).

The suspension cultures grown at $30^{\circ} \mathrm{C}$ were diluted to an $\mathrm{OD}_{730 \mathrm{~nm}}$ of 0.05 , and were further incubated at $20^{\circ} \mathrm{C}$ and $30^{\circ} \mathrm{C}$. We did not detect a significant change in the growth of WT and transgenic Synechocystis under mixotrophic cultivation at $30^{\circ} \mathrm{C}$ and $40 \mu \mathrm{mol}$ photon $/ \mathrm{m}^{2} / \mathrm{s}$ (Figure 2A), whereas growth of both WT and transgenic Synechocystis was much slower at $20^{\circ} \mathrm{C}$ than at $30^{\circ} \mathrm{C}$
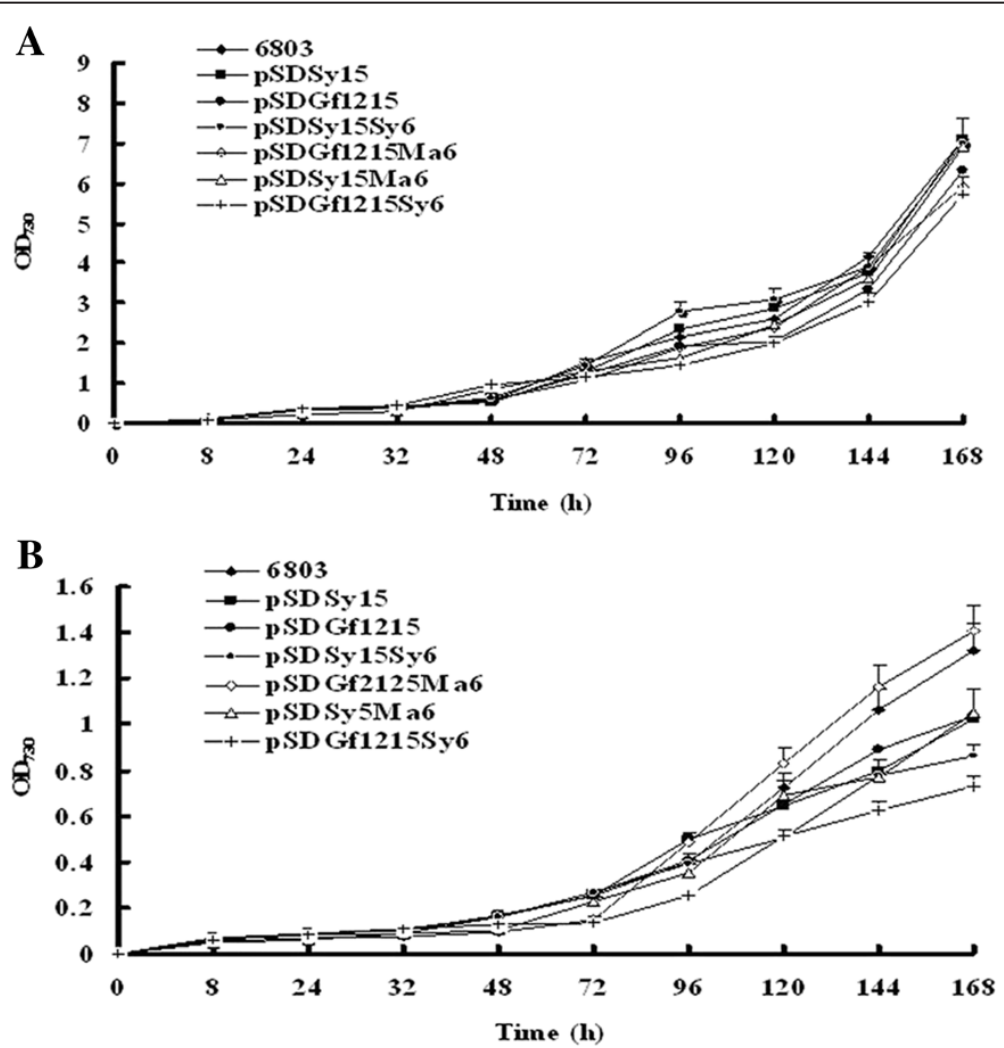

Figure 2 Growth curves of wild-type and transgenic Synechocystis. Cells were grown under mixotrophic conditions at (A) $30^{\circ} \mathrm{C}$ or (B) $20^{\circ} \mathrm{C}$. Cultures were grown in BG-11 medium and bubbled with air under an illumination of $40 \mu \mathrm{mol}$ photons $/ \mathrm{m}^{2} / \mathrm{s}$. The optical density of cells at $730 \mathrm{~nm}$ was measured at the indicated time points. Values are means \pm SD (bars) of three independent experiments conducted on different days. Absence of a bar indicates that the SD falls within the symbol. 
(Figure 2B). The growth rates of pSDGf1215Ma6 and pSDGf1215Sy6 were markedly lower than those of the WT and other transgenic Synechocystis (pSDSy15, pSDGf1215, pSDSy15Sy6, and pSDSy15Ma6) when cells were grown at $20^{\circ} \mathrm{C}$. It is unknown why overexpression of both Gf1215 and Ma6 or Sy6 affects cell growth; however, the resulting differences in lipid composition may affect the resistance of the cells to the cold. Although the transgenic Synechocystis strains had a higher ratio of $\mathrm{C} 18: 4$ at $20^{\circ} \mathrm{C}$, they grew much slower than at $30^{\circ} \mathrm{C}$. Therefore, for optimal production of PUFAs, the transgenic Synechocystis should be cultivated at $30^{\circ} \mathrm{C}$.

We found that reducing the temperature from $30^{\circ} \mathrm{C}$ to $20^{\circ} \mathrm{C}$ significantly altered FA composition in Synechocystis cells (Table 1). The reduction in temperature was accompanied by a decrease in the OA and GLA content, but an increase in LA, ALA, and SDA. Consistent with previous reports $[60,61]$, these findings indicate that $\Delta 6$, $\Delta 12$, and $\Delta 15$ desaturases are more active at $20^{\circ} \mathrm{C}$ than at $30^{\circ} \mathrm{C}$. The lower temperature did not affect the $\Delta 9$ desaturase activity, but the increase in the $\Delta 12$ desaturase activity caused an increase in LA at the expense of OA at $20^{\circ} \mathrm{C}$. Activities of both the $\Delta 6$ and $\Delta 15$ desaturases increased at $20^{\circ} \mathrm{C}$, which increased the content of ALA and SDA in both the WT and transgenic Synechocystis cells (pSDSy15, pSDSy15Sy6, and pSDSy15Ma6), and thereby enhanced production of $\omega-3$ PUFAs. The reason why GLA level decreases at $20^{\circ} \mathrm{C}$ is somewhat complex, as the increase in the $\Delta 6$ desaturase activity and LA level would be expected to increase GLA level at $20^{\circ} \mathrm{C}$. However, the decrease in GLA could be attributable, at least in part, to the competition between the $\Delta 6$ desaturases (responsible for GLA synthesis) and $\Delta 15$ desaturases (responsible for ALA synthesis) for their common substrate LA.

Biodiesel fuel derived from fats and oils has several advantages over petro-diesel. Although saturated fat is considered very suitable for the production of biodiesel, because of its oxidative stability and low levels of hydrocarbon and carbon monoxide emissions upon combustion [62], the biodiesel made from saturated fats tends to have a high melting point, and therefore, gelling of fuel may occur under cold weather, resulting in incomplete combustion and elevated mono-nitrogen oxide $\left(\mathrm{NO}_{\mathrm{x}}\right)$ emissions [63]. By contrast, biodiesel made from unsaturated fats has a lower gelling point. Therefore, unsaturated fats are an excellent source of biodiesel in cold climates [28]. With the addition of oxidative stabilizers, biodiesel made from highly unsaturated fats can be safely used as fuel.

\section{Conclusions}

In this study, we successfully constructed six homologous recombination vectors harboring genes encoding polyunsaturated FA desaturases, and expressed these genes in the blue-green alga Synechocystis sp. PCC6803 to enhance the production of ALA and SDA. We analyzed the composition and content of FAs produced under different temperatures. These results expand the current understanding of the role of FA desaturases and the molecular mechanisms that underlie PUFA accumulation at different temperatures in Synechocystis. The transgenic Synechocystis lines generated in this study produced $8.9 \mathrm{mg} / \mathrm{l}$ and $4.1 \mathrm{mg} / \mathrm{l}$ of ALA and SDA, respectively, which was more than six-fold that produced by the WT. This is the first report to show that ALA and SDA production can be increased and LA and GLA production decreased using homologous recombinants expressing endogenous and exogenous $\Delta 6$ and $\Delta 15$ FA desaturases driven by the photosynthesis $p s b A 2$ promoter. This study lays the foundation for increasing FA content in cyanobacteria and for producing large amounts of PUFAs using cyanobacteria.

\section{Methods}

\section{Strains and growth conditions}

The cyanobacterium Synechocystis sp. PCC6803 was obtained from the Freshwater Algae Culture Collection of the Institute of Hydrobiology, Chinese Academy of Sciences. Synechocystis sp. PCC6803 was cultivated in BG-11 medium (5 mM glucose) at $30^{\circ} \mathrm{C}$ [64]. For solid BG-11 medium, 1.5\% (w/v) Difco Bacto-agar (Becton Dickinson, Sparks, MD, USA), $0.3 \%(\mathrm{w} / \mathrm{v})$ sodium thiosulfate, and 10 mM TES (2-[(2-hydroxy-1, 1-bis (hydroxymethyl) ethyl) amino] ethanesulfonic acid $\mathrm{pH}$ 8.2) were added. The culture was bubbled with air under a light intensity of $40 \mu \mathrm{mol}$ photon $/ \mathrm{m}^{2} / \mathrm{s}$. Transformed strains were selected by addition of $50 \mu \mathrm{g} / \mathrm{ml}$ kanamycin (Dingguo Company, Beijing, China) to the liquid and solid BG-11 media.

\section{Genomic DNA extraction}

To extract genomic DNA from Synechocystis sp. PCC6803, $100 \mathrm{ml}$ of Synechocystis culture $\left(\mathrm{OD}_{730 \mathrm{~nm}}=1.5\right)$ was extracted with phenol:chloroform $(1: 1, \mathrm{v} / \mathrm{v})$ [65], and RNA was then removed using RNase A (TransGen Biotech, Beijing, China).

\section{Cloning of $\Delta 6$ and $\Delta 15$ genes}

The $\Delta 15$ and $\Delta 16$ genes of Synechocystis sp. PCC6803 were amplified by PCR. Genomic DNA was used as template and primers Delta 6 and Delta 15 (Table 2) were used to amplify $\Delta 6$ and $\Delta 15$, respectively. The amplified fragments of $\Delta 6$ (Sy6, $1.1 \mathrm{~kb})$ and $\Delta 15$ (Sy15, $1.1 \mathrm{~kb})$ were cloned into a pGEM-T Easy Cloning Vector (Promega, Madison, WI) and sequenced at the Biotechnology Research Center, Shandong Academy of Agricultural Sciences (Jinan, China). 
Table 2 Primers used for PCR $^{\mathrm{a}}$

\begin{tabular}{|c|c|}
\hline Primer & Sequence $5^{\prime} \rightarrow 3^{\prime}$ \\
\hline Delta 6-F & TAAGGAATTATAACCAAATGCTAACAGCGGAAAG \\
\hline Delta 6-R & $\begin{array}{l}\text { GTCCTGCAGTCAATGATGATGATGATGATGCGA } \\
\text { TGCTTTGCCCATGGCCT }\end{array}$ \\
\hline Delta 15-F & TAAGGAATTATAACCAAATGCGTCTAGAAATTTCATCG \\
\hline Delta 15-R & $\begin{array}{l}\text { CGGCTGCAGTTACTTATCGTCGTCATCCTTGTA } \\
\text { ATCAGGTITCTIITGATATC }\end{array}$ \\
\hline psbA2 promoter- $F$ & GATGTCGACGCTTTAGCGTTCCAGTG \\
\hline psbA2 promoter- $R$ & CATTTGGTTATAAT TCCTTATGTAT \\
\hline psbA2-F & СТ САTATGCCGCGGATGACAACGACTCTCCAAC \\
\hline psbA2-R & AGTGAGCTCTTAACCGTTGACAGCAGG \\
\hline
\end{tabular}

${ }^{a}$ Text that is bold and underlined indicates restriction enzyme sites (see text for details).

Optimizing $\Delta 6$ and $\Delta 15$ desaturases and gene synthesis The nucleotide sequences of $M$. alpina $\Delta 6$ FA desaturase (Ma6, GenBank: AF110510) and G. fujikuroi bifunctional $\Delta 12 / \Delta 15$ FA desaturase (Gf1215, GenBank: DQ272516) were optimized for expression in Synechocystis using the codon usage database (DNA 2.0, Menlo Park, CA, USA) and synthesized (Sangon Co., Shanghai, China).

\section{Generation of fatty acid desaturase homologous recombination plasmids containing $\Delta 6$ and $\Delta 15$ desaturase genes}

For the overexpression and heterologous expression of $\Delta 6$ and $\Delta 15$ genes (desD and desB, respectively) in Synechocystis sp. PCC6803, plasmid constructs were generated in which a His tag was added at the $3^{\prime}$ end of $\Delta 6$ and a Flag tag at the $3^{\prime}$ end of $\triangle 15$. Synechocystis psbA2 was replaced with His-tagged Synechocystis $\Delta 6$ and $M$. alpine $\Delta 6$, Flag-tagged Synechocystis $\Delta 15$, and G. fujikuroi $\Delta 12 \Delta 15$ via double homologous recombination. The $p s b A 2$ gene belongs to the $p s b A$ multi-gene family, and encodes a D1 protein of photosystem II. Mutants with inactivated $p s b A 2$ are indistinguishable from the WT [66,67]. The $p s b A 2$ promoter and open reading frame (ORF) were positioned upstream and downstream, respectively, of the FA desaturase genes in our constructs, and integrated into the shuttle vector, and the genomic psbA2 genes of the transgenic cyanobacteria were inactivated by homologous recombination. Six homologous recombination plasmids were constructed to drive the production of SDA. To achieve this, the $500 \mathrm{bp}$ fragment of Synechocystis genomic DNA upstream of the psbA2 ORF was amplified by PCR using psbA2 promoter-F and psbA2 promoter-R primers (containing a SalI site, underlined; Table 2). The $1.0 \mathrm{~kb}$ fragment of Synechocystis genomic DNA that encodes the psbA2 ORF was amplified by PCR as the downstream region of the homologous recombination vector, using the primers psbA2-F (containing NdeI and SacII sites, underlined; Table 2) and psbA2-R (containing the SacI site, underlined; Table 2). The downstream fragment was cloned into the SacII and SacI sites of pBluescript SK plus T1T2, forming plasmid pST1T2. Then, the kanamycin resistance cassette carrying npt was cloned into the single BamHI site of pST1T2, forming pSKT1T2. The upstream fragment was fused with Sy15, Gf1215, Sy6, or Ma6 by fusion PCR, and subsequently cloned into the SalI site of pSKT1T2, to form plasmids SDSy15, SDGf1215, SDSy6, and SDMa6, respectivley. SDSy6 and SDMa6 were cloned into the NdeI and SacII sites of SDSy15 and SDGf1215 to yield plasmids SDSy15Sy6, SDGf1215Ma6, SDSy15Ma6, and SDGf1215Sy6, respectivley. Six homologous recombination plasmid structures are listed (see Additional file 2: Figure S1).

\section{Transformation of Synechocystis sp. PCC6803}

The Synechocystis sp. PCC6803 strain was transformed as described by He et al. [67] and Vermaas et al. [68]. Synechocystis sp. PCC6803 was grown in liquid BG-11 medium at $30^{\circ} \mathrm{C}$ until $\mathrm{OD}_{730}$ reached 0.6 , then the cells were harvested by centrifugation and resuspended in fresh BG-11 to a density of $\mathrm{OD}_{730}=4.8$. Plasmid DNA was added to $500 \mu \mathrm{l}$ of cell suspension and mixed gently, and the mixture was incubated at $30^{\circ} \mathrm{C}$ under low light for $6 \mathrm{~h}$, and then spread on BG-11 agar plates. Transformants were selected by screening for resistance to $20 \mu \mathrm{g} /$ $\mathrm{ml}$ kanamycin. Transformants were isolated after about 10 days of incubation, and subcultured on BG-11 agar plates containing $50 \mu \mathrm{g} / \mathrm{ml}$ kanamycin. The transformants were then grown in liquid culture for analysis.

\section{SDS-PAGE and immunoblot analysis}

The crude extracts of WT and transformant cells were collected and dissolved in lysis buffer $(1 \mathrm{ml}$ of $40 \mathrm{mM}$ Tris- $\mathrm{HCl} \mathrm{pH} \mathrm{8.0)} \mathrm{with} \mathrm{protease} \mathrm{inhibitor} \mathrm{(1} \mathrm{mM} \mathrm{phenyl-}$ methanesulfonyl fluoride). After sonication, incubation, and centrifugation, the insoluble material was removed, and the supernatants were used for immunoblot analysis. The soluble proteins were separated on 12\% SDS-PAGE gels and then blotted onto 0.45- $\mu \mathrm{m}$ PVDF membranes (Beijing CoWin Biotech Co., Ltd, Beijing, China), stained with antibody to His tag or Flag tag (1:5000, Beijing CoWin Biotech Co., Ltd., Beijing, China) for $1 \mathrm{~h}$, and then treated with goat anti-rabbit IgG HRP at 1:5000 for $1 \mathrm{~h}$. Cross-reactions between protein bands and antibodies were detected using an HRP-DAB Color Development Kit (Tiangen Biotech, Beijing, China), according to the manufacturer's instructions.

\section{Lipid extraction and fatty acid methyl ester analysis of} transgenic cyanobacteria

Membrane lipid extraction from WT and genetically engineered Synechocystis sp. PCC6803 was carried out as 
described by Bligh and Dyer [69]. The colonies were collected and transferred to $1000 \mathrm{ml}$ autoclaved flasks, each containing $400 \mathrm{ml}$ of sterile BG-11 medium, and grown for 10 days at a light intensity of $40 \mu \mathrm{mol}$ photons $/ \mathrm{m}^{2} / \mathrm{s}$ and a constant temperature of $30^{\circ} \mathrm{C}$. Cultures were harvested when they reached $\mathrm{OD}_{730}=3.0$, then they were washed with distilled water and centrifuged $(6000 \times g$ for 10 minutes at room temperature) after washing to pellet cells; this washing and centrifugation was carried out three times in all. The wet cell samples were them incubated at $40^{\circ} \mathrm{C}$ to obtain $600 \mathrm{mg}$ of dry cell paste, which was diluted with $4 \mathrm{ml}$ chloroform/methanol $(1: 10 \mathrm{v} / \mathrm{v})$, then a suspension of $1 \mathrm{ml}$ hexane containing a C19:0 internal standard $(1 \mathrm{mg} / \mathrm{ml})$ was added. The mixture was heated at $80^{\circ} \mathrm{C}$ for 2 hours in a water bath, and after cooling, $5 \mathrm{ml}$ of $7 \%$ potash was added and mixed. After 10 minues of incubation at room temperature, the mixture was centrifuged at $10,000 \times g$ for 10 minutes. The supernatants (bacterial sample fatty acid methyl ester (FAME) eluate) were subjected to GC using the Elite-wax column in an ASXL instrument (Perkin-Elmer, Waltham, MA, USA). The flame-ionization detection temperature was $250^{\circ} \mathrm{C}$, and the operating temperature was maintained at $220^{\circ} \mathrm{C}$. The data are presented as the mean of three experiments for each sample.

\section{Additional files}

Additional file 1: Figure S2. Gas chromatography analysis of fatty acids (FAs) in wild-type and transgenic Synechocystis under mixotrophic conditions. The C18 FA methyl esters are labeled. We extracted the lipid from wild-type and $\Delta 6$ and $\Delta 15$ transgenic Synechocystis, which were grown at (A-D) $30^{\circ} \mathrm{C}$ or $\left(\mathbf{A}^{\prime}-\mathbf{D}^{\prime}\right) 20^{\circ} \mathrm{C}$. Strains are: $\left(\mathbf{A}\right.$ and $\left.\mathbf{A}^{\prime}\right)$ wild-type Synechocystis sp. PCC6803 (B and $\left.\mathbf{B}^{\prime}\right)$; pSDSy15; (C and $\mathbf{C}^{\prime}$ ) pSDSy15Sy6 (C and $\mathbf{C}^{\prime}$ ); and (D and $\left.D^{\prime}\right)$ pSDSy15Ma6.

Additional file 2: Figure S1. Structure of homologous recombination vectors harboring fatty acid (FA) desaturase genes. (A) SDSy15; overexpression of $\Delta 15$ FA desaturase from Synechocystis sp. PCC6803; (B) SDGf1215: overexpression of bifunctional $\Delta 12 / \Delta 15 \mathrm{FA}$ desaturase from Gibberella fujikuroi; (C) SDSy15Sy6: overexpression of $\Delta 6$ and $\Delta 15$ FA desaturase from Synechocystis sp. PCC6803; (D) SDGf1215Ma6: overexpression of $\triangle 6$ FA desaturase from Mortierella alpina and of bifunctional $\Delta 12 / \Delta 15$ FA desaturase from G. fujikuroi; (E) SDSy15Ma6: overexpression of $\triangle 6$ FA desaturase from M. alpina and of $\triangle 15 \mathrm{FA}$ desaturase from Synechocystis sp. PCC6803; and (F) SDGf1215Sy6: overexpression of $\triangle 6$ FA desaturase from Synechocystis sp. PCC6803 and of bifunctional $\triangle 12 / \Delta 15$ FA desaturase from $G$. fujikuroi, Promoter, psbA2 promoter; T1T2, rrnB (5S rRNA T1 and T2 transcription terminators from Escherichia coli); npt, neomycin phosphotransferase gene, conferring kanamycin resistance; PA2D, psbA2 open reading frame (ORF) from Synechocystis sp. PCC6803. All $\triangle 6$ FA desaturases are tailed with a His tag and all $\triangle 15$ FA desaturases are tailed with a Flag tag.

\section{Abbreviations}

ALA: a-Linolenic acid; DHA: Docosahexaenioc acid; EPA: Eicosapentaenoic acid; FA: Fatty acid; FAME: Fatty acid methyl ester; GC: Gas chromatography; GLA: Y-linolenic acid; LA: Linoleic acid; OA: Oleic acid; ORF: Open reading frame; PA: Palmitic acid; PUFAs: Polyunsaturated; PVDF: polyvinylidene difluoride; SDA: Stearidonic acid; TAG: triacylglycerol; WT: Wild-type.

\section{Competing interests}

The authors declare that they have no competing interests.

\section{Authors' contributions}

GC was responsible for study conception and design, data collection and analysis, manuscript writing and final approval of the manuscript; QW for data collection and analysis, and final approval of the manuscript; SQ, FB, YZ, $H G, J Y$, and NX for data collection and final approval of the manuscript; ZP for data analysis and final approval of the manuscript; $Y B$ for conception and data collection and final approval of the manuscript; and $\mathrm{QH}$ for conception and design, critical revision and manuscript writing, and final approval of the manuscript. All authors read and approved the final manuscript.

\section{Acknowledgements}

This work was financially supported by the International Science and Technology Cooperation Program of China (number 2012DFA30450), Shandong Province 'Taishan Scholar' Foundation (number tshw20091014), Innovation Fund for the Post-Doctoral Program of Shandong Province (number 201303029), National Natural Science Foundation of China (number 31270102), Innovation Program of the University Institutes of Jinan, Shandong Province (number 201004044) and National Science Foundation (MCB number 1120153)

\section{Author details}

${ }^{1}$ Biotechnology Research Center, Shandong Academy of Agricultural Sciences, Jinan 250100, P. R. China. ${ }^{2}$ Shandong Provincial Key Laboratory of Genetic Improvement, Ecology and Physiology of Crops, Jinan 250100, P. R. China. ${ }^{3}$ Department of Applied Science, University of Arkansas, Little Rock, Arkansas 72204, USA. ${ }^{4}$ Key Laboratory of Algal Biology, Institute of Hydrobiology, Chinese Academy of Sciences, Wuhan 430072, P. R. China. ${ }^{5}$ Test Base Service Center, Shandong Academy of Agricultural Sciences, Jinan 250100, P. R. China. ${ }^{6}$ School of Life Science, Shandong University, Jinan 250100, P. R. China.

Received: 24 July 2013 Accepted: 12 February 2014

Published: 1 March 2014

\section{References}

1. Kris-Etherton PM, Harris WS, Appel L: Fish consumption, fish oil, omega-3 fatty acids, and cardiovascular disease. Arterioscler Thromb Vasc Biol 2002, 23:2747-2757.

2. Calder PC: n-3 Fatty acids and cardiovascular disease: evidence explained and mechanisms explored. Clin Sci 2004, 107:1-11.

3. Marik PE, Varon J: Omega-3 dietary supplements and the risk of cardiovascular events: a systematic review. Clin Cardiol 2009, 32:365-372

4. Lemaitre RN, King IB, Mozaffarian D, Kuller LH, Tracy RP, Siscovick DS: n-3 polyunsaturated fatty acids, fatal ischemic heart disease, and nonfatal myocardial infarction in older adults: the cardiovascular health study. Am J Clin Nutr 2003, 77:319-325.

5. Hibbelin J: Fish consumption and major depression. Lancet 1998 351:1213.

6. Calder PC: n-3 polyunsaturated fatty acids, inflammation, and inflammatory diseases. Am J Clin Nutr 2006, 83:1505S-1519S.

7. Calder PC: PUFA, inflammatory processes and rheumatoid arthritis. Proc Nutr Soc 2008, 67:409.

8. SanGiovanni JP, Parra-Cabrera S, Colditz GA, Berkey CS, Dwyer JT: Meta-analysis of dietary essential fatty acids and long-chain polyunsaturated acids as they relate to visual resolution acuity in healthy preterm infants. Pediatrics 2000, 105:1292-1298.

9. Roynette CE, Calder PC, Dupertuis YM, Pichard C: N-3 polyunsaturated fatty acids and colon cancer prevention. Clin Nutr 2004, 23:139-151.

10. Whelan J: Dietary stearidonic acid is a long chain $(n-3)$ polyunsaturated fatty acid with potential health benefits. J Nutr 2009, 139:5-10.

11. Vázquez $L$, Akoh CC: Enrichment of stearidonic acid in modified soybean oil by low temperature crystallisation. Food Chem 2012, 130:147-155.

12. James MJ, Ursin VM, Cleland LG: Metabolism of stearidonic acid in human subjects: comparison with the metabolism of other $n-3$ fatty acids. J Nutr 2003, 77:1140-1145

13. Kawabata T, Shimoda K, Horiguchi S, Domon M, Hagiwara C, Takiyama M, Kagawa Y: Influences of stearidonic acid-enriched soybean oil on the 
blood and organ biochemical parameters in rats. Prostaglandins Leukot Essent Fatty Acids 2013, 88:179-184.

14. Venegas-Calerón M, Sayanova O, Napier JA: An alternative to fish oils: metabolic engineering of oil-seed crops to produce omega-3 long chain polyunsaturated fatty acids. Prog Lipid Res 2010, 49:108-119.

15. Cordain L, Eaton SB, Sebastian A, Mann N, Lindeberg S, Watkins BA, O'Keefe $\mathrm{J}$, Brand-Miller J: Origins and evolution of the Western diet: health implications for the 21st century. Am J Clin Nutr 2005, 81:341-354.

16. Simopoulos AP: Essential fatty acids in health and chronic disease. Am J Clin Nutr 1999, 70:560S-569S

17. Ueshima H, Stamler J, Elliott P, Chan Q, Brown IJ, Carnethon MR, Daviglus ML, He K, Moag-Stahlberg A, Rodriguez BL, Steffen LM, van Horn L, Yarnell J, Zhou B: Food omega-3 fatty acid intake of individuals (total, linolenic acid, long-chain) and their blood pressure: INTERMAP study. Hypertension 2007, 50:313-319.

18. von Schacky C: A review of omega-3 ethyl esters for cardiovascular prevention and treatment of increased blood triglyceride levels. Vasc Health Risk Manag 2006, 2:251-262.

19. Kremer JM, Lawrence DA, Petrillo GF, Litts LL, Mullaly PM, Rynes RI, Stocker RP, Parhami N, Greenstein NS, Fuchs BR: Effects of high-dose fish oil on rheumatoid arthritis after stopping nonsteroidal antiinflammatory drugs clinical and immune correlates. Arthritis Rheum 1995, 38:1107-1114.

20. Nagel G, Nieters A, Becker N, Linseisen J: The influence of the dietary intake of fatty acids and antioxidants on hay fever in adults. Allergy 2003, 58:1277-1284.

21. Simopoulos AP: Omega-3 fatty acids in inflammation and autoimmune diseases. J Am Coll Nutr 2002, 21:495-505.

22. Simopoulos AP: The importance of the ratio of omega- $6 /$ omega- 3 essential fatty acids. Biomed Pharmacother 2002, 56:365-379.

23. Nugent AP: The metabolic syndrome. Nutr Bull 2004, 29:36-43.

24. Roessler PG, Brown LM, Dunahay TG, Heacox DA, Jarvis EE, Schneider JC, Talbot SG, Zeiler KG: Genetic engineering approaches for enhanced production of biodiesel fuel from microalgae. ACS Symp Ser 1994, 566:255-270.

25. Dunahay TG, Jarvis EE, Dais SS, Roessler PG: Manipulation of microalgal lipid production using genetic engineering. App/ Biochem Biotechnol 1996, 57:223-231

26. Chisti Y: Biodiesel from microalgae. Biotechnol Adv 2007, 25:294-306.

27. Bouaid A, Martinez M, Aracil J: Long storage stability of biodiesel from vegetable and used frying oils. Fuel 2007, 86:2596-2602.

28. Gui MM, Lee KT, Bhatia S: Feasibility of edible oil vs. non-edible oil vs. waste edible oil as biodiesel feedstock. Energy 2008, 33:1646-1653.

29. Cunnane SC: Problems with essential fatty acids: time for a new paradigm? Prog Lipid Res 2003, 42:544-568.

30. Hibbeln JR, Nieminen LR, Blasbalg TL, Riggs JA, Lands WE: Healthy intakes of n-3 and n-6 fatty acids: estimations considering worldwide diversity. Am J Clin Nutr 2006, 83:1483S-1493S.

31. Pauly $D$, Watson $R$, Alder J: Global trends in world fisheries: impacts on marine ecosystems and food security. Philos Trans R Soc London B Biol SCi 2005, 360:5-12.

32. Drexler H, Spiekermann P, Meyer A, Domergue F, Zank T, Sperling P, Abbadi A, Heinz E: Metabolic engineering of fatty acids for breeding of new oilseed crops: strategies, problems and first results. J Plant Physio/ 2003, 16:779-802.

33. Robert SS: Production of eicosapentaenoic and docosahexaenoic acidcontaining oils in transgenic land plants for human and aquaculture nutrition. Mar Biotechnol 2006, 8:103-109.

34. Damude HG, Kinney AJ: Enhancing plant seed oils for human nutrition. Plant Physiol 2008, 147:962-968.

35. Gill I, Valiveyt R: Polyunsaturated fatty acids, Part I: occurrence, biological activities and applications. Trends Biotehnol 1997, 15:401-409.

36. Napier JA: The production of unusual fatty acids in transgenic plants. Annu Rev Plant Biol 2007, 58:295-319.

37. Radakovits R, Jinkerson RE, Darzins A, Posewitz MC: Genetic engineering of algae for enhanced biofuel production. Eukaryot Cell 2010, 9:486-501.

38. Mutanda T, Ramesh D, Karthikeyan S, Kumari S, Anandraj A, Bux F: Bioprospecting for hyper-lipid producing microalgal strains for sustainable biofuel production. Bioresour Technol 2011, 102:57-70.

39. Lei A, Chen H, Shen G, Hu Z, Chen L, Wang J: Expression of fatty acid synthesis genes and fatty acid accumulation in Haematococcus pluvialis under different stressors. Biotechnol Biofuels 2012, 5:1-11.
40. Harwood $J$, Guschina IA: The versatility of algae and their lipid metabolism. Biochimie 2009, 91:679-684.

41. Riekhof WR, Sears BB, Benning C: Annotation of genes involved in glycerolipid biosynthesis in Chlamydomonas reinhardtii: discovery of the betaine lipid synthase BTA1Cr. Eukaryot Cell 2005, 4:242-252.

42. Napier JA, Beaudoin F, Michaelson LV, Sayanova O: The production of long chain polyunsaturated fatty acids in transgenic plants by reverse engineering. Biochimie 2004, 86:785-792.

43. Sayanova OV, Napier JA: Eicosapentaenoic acid: biosynthetic routes and the potential for synthesis in transgenic plants. Phytochemistry 2004, 65:147-158

44. Novikova GV, Moshkov IE, Los DA: Proteinsensors and transducers of cold and osmotic stress in cyanobacteria and plants. Mol Biol (Moscow) 2007, 41:427-437

45. Los DA, Zinchenko W: Regulatory role of membrane fluidity in gene expression. Lipids Photosynth Springer Neth 2010, 30:329-348.

46. Mansilla MC, Cybulski LE, Albanesi D, de Mendoza D: Control of membrane lipid fluidity bymolecular thermosensors. J Bacterio/ 2004 186:6681-6688.

47. Mironov KS, Maksimov EG, Maksimov GV, Los DA: Feedback between fluidity of membranes and transcription of the desB gene for the $\omega 3$-desaturase in the cyanobacterium Synechocystis. Mol Biol 2012, 46:134-141.

48. Damude HG, Zhang HX, Farrall L, Ripp KG, Tomb JF, Hollerbach D, Yadav NS: Identification of bifunctional $\Delta 12 / \omega 3$ fatty acid desaturases for improving the ratio of $\omega 3$ to $\omega 6$ fatty acids in microbes and plants. Proc Natl Acad Sci U S A 2006, 103:9446-9451.

49. Reddy AS, Thomas TL: Expression of a cyanobacterial D6-desaturase gene results in gamma-linolenic acid production in transgenic plants. Nat Biotechnol 1996, 14:639-642.

50. Sayanova O, Smith MA, Lapinskas P, Stobart AK, Dobson G, Christie WW, Shewry PR, Napier JA: Expression of a borage desaturase CDNA containing an $\mathrm{N}$-terminal cytochrome b5 domain results in the accumulation of high levels of $\Delta 6$-desaturated fatty acids in transgenic tobacco. Proc Natl Acad Sci U S A 1997, 94:4211-4216.

51. Qiu X, Hong H, Datla N, MacKenzie SL, Taylor DC, Thomas TL: Expression of Borage $\Delta 6$-desaturase in Saccharomyces cerevisiae and oilseed crops. Can J Bot 2002, 80:42-49.

52. Hammond BG, Lemen JK, Ahmed G, Miller KD, Kirkpatrick J, Fleeman T: Safety assessment of SDA soybean oil: results of a 28-day gavage study and a 90-day/one generation reproduction feeding study in rats. Regul Toxicol Pharmacol 2008, 52:311-323.

53. Ruiz-López N, Haslam RP, Venegas-Calerón M, Larson TR, Graham IA, Napier JA, Sayanova O: The synthesis and accumulation of stearidonic acid in transgenic plants: a novel source of 'heart-healthy' omega-3 fatty acids. Plant Biotechnol J 2009, 63:704-716.

54. Ruiz-López N, Sayanova O, Napier JA, Haslam RP: Metabolic engineering of the omega-3 long chain polyunsaturated fatty acid biosynthetic pathway into transgenic plants. J Exp Bot 2012, 63:2397-2410

55. Guedes A, Amaro HM, Barbosa CR, Pereira RD, Malcata FX: Fatty acid composition of several wild microalgae and cyanobacteria, with a focus on eicosapentaenoic, docosahexaenoic and a-linolenic acids for eventual dietary uses. Food Res Int 2011, 44:2721-2729.

56. Chen R, Matsui K, Ogawa M, Oe M, Ochiai M, Kawashima H, Sakuradani E, Shimizu S, Ishimoto M, Hayashi M, Murooka Y, Tanaka Y: Expression of $\Delta 6$, $\Delta 5$ desaturase and GLELO elongase genes from Mortierella alpine for production of arachidonic acid in soybean [Glycine max (L.) Merrill] seeds. Plant Sci 2006, 170:399-406.

57. Satu N, Murata N: Temperature shift-induced responses in lipids in the blue-green alga, Anabaena variabilis: the central role of diacylmonoalactosylglycerol in thermo adaption. Biochim Biophys Acta 1980, 619:353-365.

58. Renaud SM, Thinh LV, Lambrinidis G, Parry DL: Effect of temperature on growth, chemical composition and fatty acid composition of tropical Australian microalgae grown in batch cultures. Aquaculture 2002, 211:195-214.

59. Madigan MT, Martinko JM, Dunlap PV, Clark DP: Brock Biology of Microorganisms, 12th edn. Int Microbiol 2008, 11:65-73.

60. Los DA, Murata N: Low-temperature induced accumulation of the desaturase gene transcript in Synechocystis PCC 6803 results from both acceleration of transcription and increase in mRNA stability. Russian $J$ Plant Physiol 1994, 41:147-151. 
61. Wada H, Murata N: Temperature-induced changes in the fatty acids composition of the cyanobacterium, Synechocystis PCC 6803. Plant Physiol 1990, 92:1062-1069.

62. Atadashi IM, Aroua MK: Abdul Aziz AR, Sulaiman NMN: production of biodiesel using high free fatty acid feedstocks. Renew Sust Energ Rev 2012, 16:3275-3285

63. Boehman AL: Biodiesel production and processing: foreword. Fuel Process Technol 2005, 86:1057-1058.

64. Stanier RY, Kunisawa R, Mandel M, Cohen-Bazire G: Purification and properties of unicellular blue-green algae (order Chroococcales). Bacteriol Rev 1971, 35:171-205.

65. Nakamura Y, Kaneko T, Hirosawa M, Miyajima N, Tabata S: CyanoBase, a www database containing the complete nucleotide sequence of the genome of Synechocystis sp. strain PCC6803. Nucleic Acids Res 1998, 26:63-67.

66. Mohamed A, Eriksson J, Osiewacz HD, Jansson C: Differential expression of the psbA genes in the cyanobacterium Synechocystis 6803. Mol Gen Genet 1993, 238:161-168.

67. Qingfang $H$, Schlich T, Paulsen $H$, Vermass W: Expression of a higher plant light-harvesting cholrophy $11 \mathrm{a} / \mathrm{b}$-binding proterin in Synechocystis sp. PCC6803. Eur J Biochem 1999, 263:561-570.

68. Vermaas WFJ, Williams JGK, Arntzen CJ: Sequencing and modification of $\mathrm{psbB}$, the gene encoding the CP-47 protein of photosystem II, in the cyanobacterium Synechocystis 6803. Plant Mol Biol 1987, 8:317-326.

69. Bligh EG, Dyer WJ: A rapid method of total lipid extraction and purification. Can J Biochem Physiol 1959, 37:911-917.

doi:10.1186/1754-6834-7-32

Cite this article as: Chen et al:: Transgenic expression of delta- 6 and delta- 15 fatty acid desaturases enhances omega- 3 polyunsaturated fatty acid accumulation in Synechocystis sp. PCC6803. Biotechnology for Biofuels 2014 7:32.

\section{Submit your next manuscript to BioMed Central and take full advantage of:}

- Convenient online submission

- Thorough peer review

- No space constraints or color figure charges

- Immediate publication on acceptance

- Inclusion in PubMed, CAS, Scopus and Google Scholar

- Research which is freely available for redistribution 